\title{
A Numeric-Symbolic Solution of GNSS Phase Ambiguity
}

\author{
Béla Paláncz ${ }^{1 *}$, Lajos Völgyesi \\ 1 Department of Photogrammetry and Geoinformatics, Faculty of Civil Engineering, Budapest University of Technology and \\ Economics, H-1521 Budapest, P.O.B. 91, Hungary \\ 2 Department of Geodesy and Surveying, Faculty of Civil Engineering, Budapest University of Technology and Economics, H-1521 \\ Budapest, P.O.B. 91, Hungary \\ * Corresponding author, e-mail: palancz@epito.bme.hu
}

Received: 11 October 2019, Accepted: 18 November 2019, Published online: 03 February 2020

\begin{abstract}
Solution of the Global Navigation Satellite Systems (GNSS) phase ambiguity is considered as a global quadratic mixed integer programming task, which can be transformed into a pure integer problem with a given digit of accuracy. In this paper, three alternative algorithms are suggested. Two of them are based on local and global linearization via McCormic Envelopes, respectively. These algorithms can be effective in case of simple configuration and relatively modest number of satellites. The third method is a locally nonlinear, iterative algorithm handling the problem as $\{-1,0,1\}$ programming and also lets compute the next best integer solution easily. However, it should keep in mind that the algorithm is a heuristic one, which does not guarantee to find the global integer optimum always exactly. The procedure is very powerful utilizing the ability of the numeric-symbolic abilities of a computer algebraic system, like Wolfram Mathematica and it is properly fast for minimum 4 satellites with normal configuration, which means the Geometric Dilution of Precision (GDOP) should be between 1 and 8. Wolfram Alpha and Wolfram Clouds Apps give possibility to run the suggested code even via cell phones. All of these algorithms are illustrated with numerical examples. The result of the third one was successfully compared with the LAMBDA method, in case of ten satellites sending signals on two carrier frequencies (L1 and L2) with weighting matrix used to weight the GNSS observation and computed as the inverse of the corresponding covariance matrix.
\end{abstract}

Keywords

GNSS, GPS cycle ambiguities, computer algebra, local and global linearization, successive nonlinear method, mixed integer programming

\section{Introduction}

Highly accurate static Global Navigation Satellite Systems (GNSS) positioning is achieved by the processing of relative phase ranges observed to the GNSS satellites at both the reference and the rover stations [1,2]. To eliminate common biases such as the satellite and receiver clock error, the double-differenced phase observations are formed and they are adjusted using a least squares adjustment. The linearized observation equation of the double-differenced phase observations has the following form, see [3].

$\Delta \Delta \Phi_{A B}^{j k}=a_{1} \delta x_{B}^{j k}+a_{2} \delta y_{B}^{j k}+a_{3} \delta z_{B}^{j k}+\lambda \delta N_{A B}^{j k}$,

where $\Delta \Delta \Phi_{A B}^{j k}$ is the double differences phase observations taken to the $j$-th and $k$-th satellite, $\delta x_{B}, \delta y_{B}$ and $\delta z_{B}$ are the relative coordinate differences between the reference (A) and rover (B) stations, $\lambda$ is the wavelength of the signal, $\delta N_{A B}^{j k}$ is the double differenced phase ambiguity and $j$ refers to the so-called pivot satellite, that is used as a reference for forming the double differences.
The terms $a_{i}$ in Eq. (1) stand for the coefficients resulted from the partial derivates of the linearized geometrical pseudorange distant equations. Let us assume that five satellites are measured concurrently on both the reference and the rover stations in two consecutive epochs. Since one satellite is used as a pivot satellite, four double differences are formed in each epoch. This means that altogether 8 observation equations are formed, which can be used to evaluate 7 unknowns (3 coordinate differences and 4 double-differenced phase ambiguities).

A usual solution of the problem is to estimate the unknowns using a least-squares adjustment, where the phase ambiguities are integers, while the coordinates are floating point variables. Consequently, the computation of the integer least-squares estimates of the GNSS cycle ambiguities leads to a mixed integer quadratic problem see $[4,5]$, $(y-A x-B z)^{T} Q_{y}^{-1}(y-A x-B z) \rightarrow \min _{x, z}$, 
where $y$ vector of double differences carrier phase observation in cycles, $A$ design matrix for continuous-valued parameters (baseline components), $B$ design matrix for ambiguities, $x$ is unknown vector of continuous parameters, $\mathrm{x} \in \mathcal{R}^{3}, z$ the unknown ambiguity vector in cycles, $z \in Z^{m}$, where $m$ depends on the number of the satellites and the carrier frequencies. The matrix $Q_{y}^{-1}$ is the weight matrix $\left(Q_{y}\right.$ is the covariance matrix).

Solving the problem Eq. (2) is well known to be NP hard. In other words, there exists no algorithm to find the global optimal integer solution to the problem Eq. (2) in polynomial time, see, [6]. Thus, for real time applications such as wireless communication and Global Positioning Systems (GPS) kinematic positioning with many integer ambiguities due to the use of different wavelengths and/or different navigation satellite systems, it may be more realistic to expect some good suboptimal integer solutions than to find the global optimal integer solution. Basically, all the methods to construct suboptimal integer solutions may be classified into two types: simple rounding and sequential rounding. This is the whole point of the development of the LAMBDA approach by Teunissen et al in the 1990s, i.e. [7]. LAMBDA does not "solve" integer rounding or sequential rounding it is a tool to make ILS more efficient. To solve this task, probably the most popular procedure is the so-called LAMBDA method, see $[8,9]$.

In this article, three different methods are introduced to solve quadratic integer programming: local linearization, global linearization and sequential nonlinear approach. All these methods can be time-wise effective in case of simple configuration and relatively low number of satellites (less than 8-10). The third method is utilizing the ability of the numeric-symbolic abilities of a computer algebraic system, like Wolfram Mathematica and properly fast for normal satellite configuration. Wolfram Alpha and Wolfram Clouds Apps give possibility to run the suggested code via cell phones.

In the first part the three methods to solve quadratic integer programming are introduced and illustrated via a simple example. Then the third method is demonstrated for different satellite configurations: a simple one with one carrier frequency using synthetic data with two different carrier frequencies based on real field measured data, provided by Khodabandeh [10]. The results are compared with those of the latest version of the LAMBDA method.

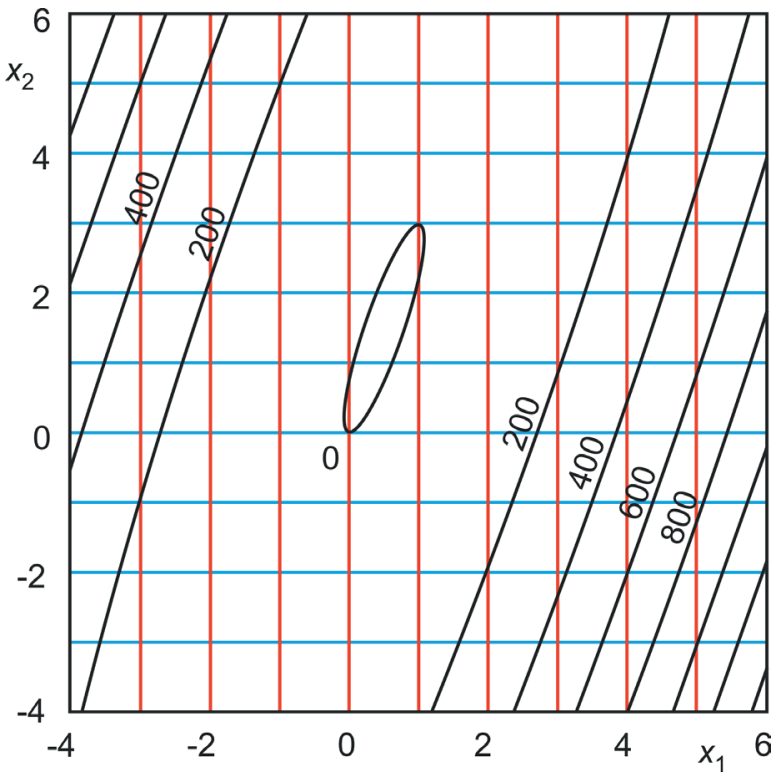

Fig. 1 Contour plot of the objective function

\section{Three methods to solve integer programming}

In Section 2, three different methods are discussed and illustrated. All of them are based on the global float (floating point) solution of the problem. Let us consider a simple integer quadratic programming example adapted from $\mathrm{Li}$ and Sun [11]. We should minimize the following objective function,

$q=27 x_{1}^{2}-18 x_{1} x_{2}+4 x_{2}^{2}-3 x_{2}$.

Let us visualize the problem, see Fig. 1.

We have to remark that this toy-problem can be solved directly. Excluding trivial solution $\left(x_{1}=0, x_{2}=0\right)$, the integer minimum of $q\left(x_{1}, x_{2}\right)$ is $\left.x_{1}=1, x_{2}=3\right)$.

However, employing linearization the computation time can be reduced considerably, see later.

\subsection{Local linearization}

Let us linearize this $q\left(x_{1}, x_{2}\right)$ function around at the point of $\left\{x_{10}, x_{20}\right\}$ :

$$
\begin{aligned}
& q L=27 x_{10}^{2}+\left(x_{1}-x_{10}\right)\left(54 x_{10}-18 x_{20}\right)-3 x_{20} \\
& -18 x_{10} x_{20}+4 x_{20}^{2}+\left(x_{2}-x_{20}\right)\left(-3-18 x_{10}+8 x_{20}\right) .
\end{aligned}
$$

The float minimum of $q\left(x_{1}, x_{2}\right)$ is $\left(x_{1}=0.5, x_{2}=1.5\right)$. Then the linearization point can be, $x_{10}=1, x_{20}=2$.

Then linearized model is,

$$
q L_{0}=-7+18 x_{1}-5 x_{2} .
$$


To minimize Eq. (5), constraints are required, here we use simply a heuristic approach suggested by Champton and Strzebonski [12], assuming, that $x_{i 0}-1 \leq x_{i} \leq x_{i 0}+1$, $i=1,2$. Therefore, let us introduce new variables $\mu_{i}=x_{i}-x_{i 0}$ to get a $(-1,0,1)$ linear programming problem. Then

$q L_{0 \mu}=1+18 \mu_{1}-5 \mu_{2}$.

The lower and upper bounds for the variables are $\left\{-1 \leq \mu_{1} \leq 1,-1 \leq \mu_{2} \leq 1\right\}$.

This linear problem can be solved via linear programming. It can be written in the form of $\min c \mu$ under the restriction $m \mu \geq b$, where

$$
c=(18,-5), \quad m=\left(\begin{array}{rr}
1 & 0 \\
0 & 1 \\
-1 & 0 \\
0 & -1
\end{array}\right), \quad b=\left(\begin{array}{l}
-1 \\
-1 \\
-1 \\
-1
\end{array}\right)
$$

The solution is $\Delta=\{0,1\}$, then $x_{0}=x_{0}+\Delta=\{1,3\}$. Employing this result a new linearization point is $x_{0}=\{1,3\}$.

Since $\{1,2\} \rightarrow\{1,3\}$, the minimum is at $\{1,3\}$. The running time is considerably smaller than it was in case of the global nonlinear solution.

\subsection{Global linearization}

In this case linearization is carried out not around a single point but on a restricted domain. The global bound is the domain where the integer solution may exist, and its center is the float solution.

\subsubsection{Global bound}

The radius of this domain can be computed from the ratio of the maximal and minimal eigenvalues of the following bilinear form [11]. This bilinear form in our case is

$$
\left(\begin{array}{ll}
\tilde{x}_{1}-0.5 & \tilde{x}_{2}-1.5
\end{array}\right) Q\left(\begin{array}{c}
\tilde{x}_{1}-0.5 \\
\tilde{x}_{2}-1.5
\end{array}\right),
$$

where $\tilde{x}_{1}$ and $\tilde{x}_{2}$ are the integer solutions of the optimization problem and the center of this domain is the float solution $\left\{x_{1}=0.5, x_{2}=1.5\right\}$. The matrix $Q$ can be computed as

$$
Q=\frac{1}{2}\left(\begin{array}{cc}
\frac{\partial^{2} q}{\partial x_{1}^{2}} & \frac{\partial^{2} q}{\partial x_{1} \partial x_{2}} \\
\frac{\partial^{2} q}{\partial x_{1} \partial x_{2}} & \frac{\partial^{2} q}{\partial x_{2}^{2}}
\end{array}\right) .
$$

Then the matrix of the bilinear form in our case is $Q=\left(\begin{array}{cc}27 & -9 \\ -9 & 4\end{array}\right)$ and the eigenvalues $\{30.1031,0.896918\}$.

The ratio of the maximum and minimum values of $\lambda$ 's is $\kappa=33.5628$. Then the radius of a $\mathrm{n}$ dimensional hypersphere with the float solution as a centre, $R=1 / 2 \sqrt{n \kappa}$, now $n=2$, so $R=4.09651$.

Using box-type constraint

$-3 \leq x_{1} \leq 4,-2 \leq x_{2} \leq 5$.

Box-bounded region can be seen in Fig. 2.

Having global bound for the solution, the problem becomes a constrained nonlinear problem. Further simplification is possible via linearization of the objective function. In order to linearize our function over this region, McCormick Envelopes is employed, which is described in the next paragraph.

\subsubsection{Linearization via McCormick envelopes}

The McCormick envelopes are the convex relaxation of a quadratic problem via introducing new variables for the quadratic terms and employing the additional constraints [13]. In general, we introduce new variables,

$$
w_{i j}=x_{i} x_{j},
$$

with following constraints,

$$
\begin{aligned}
& w_{i j} \geq x_{i L} x_{j}+x_{i} x_{j L}-x_{i L} x_{j L} \\
& w_{i j} \geq x_{i U} x_{j}+x_{i} x_{j U}-x_{i U} x_{j U} \\
& w_{i j} \leq x_{i U} x_{j}+x_{j L} x_{i}-x_{i U} x_{j L} \\
& w_{i j} \leq x_{i} x_{j U}+x_{i L} x_{j}-x_{j U} x_{i L}
\end{aligned}
$$

where $x_{L} \leq x \leq x_{U}$.

Let us employ McCormic envelopes approach to our simple quadratic problem. Employing Eq. (11), the linear objective function of our example is,

$q L=27 w_{11}-18 w_{12}+4 w_{22}-3 x_{2}$.

The box-type bounds are,

$$
x_{1 L}=-3 ; x_{1 U}=4 ; x_{2 L}=-2 ; x_{2 U}=5 \text {, }
$$

and the function values at the lower and upper bounds are 157.

The additional inequality constraints, the McCormick envelopes are, 


$$
\begin{aligned}
& w_{11} \geq x_{1 L} x_{1}+x_{1} x_{1 L}-x_{1 L} x_{1 L}, \\
& w_{11} \geq x_{1 U} x_{1}+x_{1} x_{1 U}-x_{1 U} x_{1 U}, \\
& w_{11} \leq x_{1 U} x_{1}+x_{1} x_{1 L}-x_{1 U} x_{1 L}, \\
& w_{11} \leq x_{1} x_{1 U}+x_{1 L} x_{1}-x_{1 U} x_{1 L}, \\
& w_{22} \geq x_{2 L} x_{2}+x_{2} x_{2 L}-x_{2 L} x_{2 L}, \\
& w_{22} \geq x_{2 U} x_{2}+x_{2} x_{2 U}-x_{2 U} x_{2 U}, \\
& w_{22} \leq x_{2 U} x_{2}+x_{2} x_{2 L}-x_{2 U} x_{2 L}, \\
& w_{12} \geq x_{1 L} x_{2}+x_{1} x_{2 L}-x_{1 L} x_{2 L}, \\
& w_{12} \geq x_{1 U} x_{2}+x_{1} x_{2 U}-x_{1 U} x_{2 U}, \\
& w_{12} \leq x_{1 U} x_{2}+x_{1} x_{2 L}-x_{1 U} x_{2 L}, \\
& w_{12} \leq x_{1} x_{2 U}+x_{1 L} x_{2}-x_{2 U} x_{1 L}, \\
& w_{11}>0, w_{12} \neq 0, \quad w_{22}>0, \\
& x_{1 L} \leq x_{1} \leq x_{1 U}, x_{2 L} \leq x_{2} \leq x_{2 U}, x_{1} \neq 0, x_{2} \neq 0
\end{aligned}
$$

\section{therefore}

$w_{11} \geq-9-6 x_{1}, w_{11} \geq-16+8 x_{1} . w_{11} \leq 12+x_{1}$,

$w_{11} \leq 12+x_{1}, \quad w_{22} \geq-4-4 x_{2}, \quad w_{22} \geq-25+10 x_{2}$,

$w_{22} \leq 10+3 x_{2}, w_{12} \geq-6-2 x_{1}-3 x_{2}$,

$w_{12} \geq-20+5 x_{1}+4 x_{2}, \quad w_{12} \leq 8-2 x_{1}+4 x_{2}$,

$w_{12} \leq 15+5 x_{1}-3 x_{2}, w_{11}>0, w_{12} \neq 0, w_{22}>0$,

$-3 \leq x_{1} \leq 4,-2 \leq x_{2} \leq 5, x_{1} \neq 0, x_{2} \neq 0$,

$\left(x_{1}\left|x_{2}\right| w_{11}\left|w_{12}\right| w_{22}\right) \in$ Integers.

Now, this is a linear integer programming problem. The price of the linearization is the increase in the number of variables.

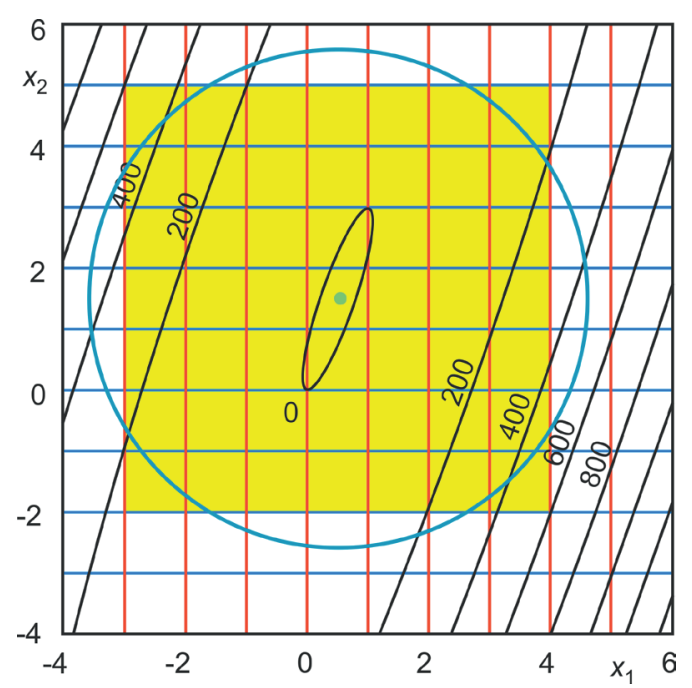

Fig. 2 Disk and the box-bounded region of the global integer optimum
Considering the new variables $\left(x_{1}, x_{2}, w_{11}, w_{12}, w_{22}\right)$, in Eq. (13), the coefficient vector of the objective function is $c=\{0,-3,27,-18,4\}$.

We introduce a small positive constant $\varepsilon=10^{-3}$ in order to exclude the trivial solution $\{0,0\}$. Then the constraints are,

$$
m=\left(\begin{array}{ccccc}
-2 x_{1 L} & 0 & 1 & 0 & 0 \\
-2 x_{1 U} & 0 & 1 & 0 & 0 \\
x_{1 U}+x_{1 L} & 0 & -1 & 0 & 0 \\
0 & -2 x_{2 L} & 0 & 0 & 1 \\
0 & -2 x_{2 U} & 0 & 0 & 1 \\
0 & x_{2 U}+x_{2 L} & 0 & 0 & -1 \\
-x_{2 L} & -x_{1 L} & 0 & 1 & 0 \\
-x_{2 U} & -x_{1 U} & 0 & 1 & 0 \\
x_{2 L} & x_{1 U} & 0 & -1 & 0 \\
x_{2 U} & x_{1 L} & 0 & -1 & 0 \\
0 & 0 & 1 & 0 & 0 \\
0 & 0 & 0 & 1 & 0 \\
0 & 0 & 0 & 0 & 1 \\
1 & 0 & 0 & 0 & 0 \\
1 & 0 & 0 & 0 & 0 \\
0 & 1 & 0 & 0 & 0 \\
0 & 1 & 0 & 0 & 0 \\
1 & 0 & 0 & 0 & 0 \\
0 & 1 & 0 & 0 & 0
\end{array}\right) ; \quad b=\left(\begin{array}{c}
-x_{1 L} x_{1 L} \\
-x_{1 U} x_{1 U} \\
x_{1 U} x_{1 L} \\
-x_{2 L} x_{2 L} \\
-x_{2 U} x_{2 U} \\
x_{2 U} x_{2 L} \\
-x_{1 L} x_{2 L} \\
-x_{1 U} x_{2 U} \\
x_{1 U} x_{2 L} \\
x_{2 U} x_{1 L} \\
\epsilon \\
\epsilon \\
\epsilon \\
x_{1 L} \\
-x_{1 U} \\
x_{2 L} \\
-x_{2 U} \\
\epsilon \\
\epsilon
\end{array}\right)
$$

Now linear programming can be employed, which result is $\left\{x_{1}, x_{2}\right\}=\{2,3\}$. Using this result as a new upper limit, see Fig. 3, the second approach can be computed,

$x_{1 L}=-3 ; x_{1 U}=2 ; x_{2 L}=-2 ; x_{2 U}=3$,

and accordingly, a new McCormick envelopes will be determined. The value of the objective function at the lower bound is 157 and at the upper bound is 27. The results of this iteration process can be seen in Table 1 .

Table 1 Results of the global linearization

\begin{tabular}{lccccc}
\hline Iteration & $\begin{array}{c}\text { Bounds } \\
\text { for } x_{1}\end{array}$ & $\begin{array}{c}\text { Bounds } \\
\text { for } x_{2}\end{array}$ & Solution & $\begin{array}{c}\text { Objective } \\
\text { at lower } \\
\text { bound }\end{array}$ & $\begin{array}{c}\text { Objective } \\
\text { at upper } \\
\text { bound }\end{array}$ \\
\hline 0 & $\{-3,4\}$ & $\{-2,5\}$ & $\{2,3\}$ & 157 & 157 \\
1 & $\{-3,2\}$ & $\{-2,3\}$ & $\{1,2\}$ & 157 & 27 \\
2 & $\{1,2\}$ & $\{2,3\}$ & $\{1,3\}$ & 1 & 27 \\
3 & $\{1,1\}$ & $\{3,3\}$ & $\{1,3\}$ & 0 & 0 \\
\hline
\end{tabular}




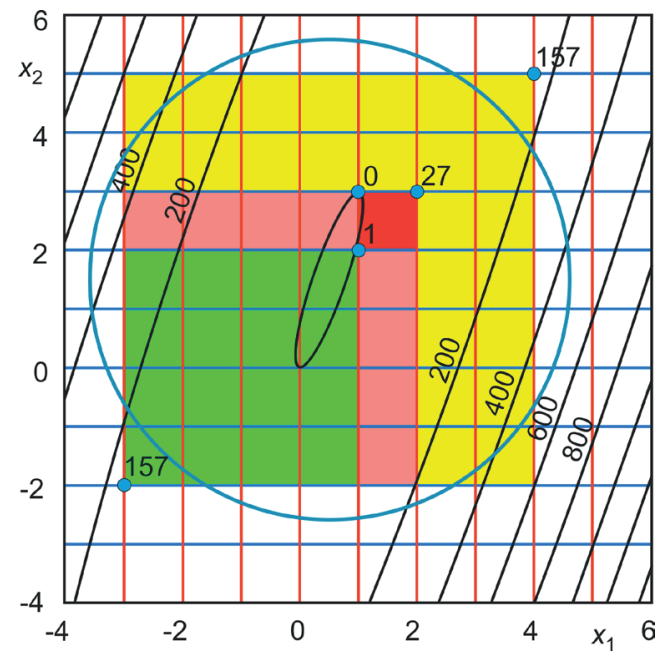

Fig. 3 Box-regions of the first three iterations of the linear problem. The meaning of the values of the box-regions can be seen in Table 1

No more approximation step is necessary since the next iteration will give the same result, so it is a fixed point of the iteration process, see Fig. 3.

This method is converging, however now the size of the linear model is $19 \times 5$. After the linearization techniques, in the next Section 2.3 a non-linear method will be discussed.

\subsection{Successive nonlinear method}

Here we employ the heuristic technique suggested by [12]. We are looking for an improved integer solution $x_{i}+1$ in the neighborhood $\mathcal{N}$ of the actual one, $x_{i}$, assuming that $x_{i+1} \in \mathcal{N}\left(x_{i}: L_{1}\left(x_{i}, x_{i+1}\right)=1\right)$ that $x_{i}+1$ is in the neighborhood of $x_{i}$ with $L_{1}$ norm equal 1 . In this way, we have a $\{-1,0,1\}$ quadratic problem.

Starting with $x_{0}=\{1,2\}$, and introducing the new variables $x_{1}=x_{01}+\mu_{1}$ and $x_{2}=x_{02}+\mu_{2}$ we get our objective function

$q=1+18 \mu_{1}+27 \mu_{1}^{2}-5 \mu_{2}-18 \mu_{1} \mu_{2}+4 \mu_{2}^{2}$,

and the constraints are: $-1 \leq \mu_{1} \leq 1,-1 \leq \mu_{2} \leq 1$.

Then minimizing $q$, the solution can be computed,

$x_{0}=\{1,2\}+\{0,1\}=\{1,3\}$.

Now no more computation step is necessary.

Until now, we have considered a pure integer problem. However, in the case of mixed integer problem, a part of the variables are continuous variables.

\section{Mixed integer programming}

This type of the problem can be transformed into a pure integer problem. Let us consider the following illustrative example. Let the function to be maximized is, $u=3 x_{1}+5 x_{2}+y_{1}+2 y_{2}$,

with continuous or "float" variables $\left(y_{1}, y_{2}\right) \in \mathcal{R}$, and with integer variables $\left(x_{1}, x_{2}\right) \in \mathcal{Z}$. First, let us solve the continuous version of the problem. The constraints are

$2 x_{1}+x_{2}+y_{1}+2 y_{2} \leq 4 ; \quad x_{1}+3 x_{2}+y_{1}+y_{2} \leq 5 ;$

$x_{1} \geq 0 ; \quad x_{2} \geq 0 ; \quad y_{1} \geq 0 ; \quad y_{2} \geq 0$.

Then the continuous solution is (employing post rationalization),

$x_{1}=7 / 5, x_{2}=6 / 5$.

Now, we introduce new integer variables as

$\xi_{i}=10^{\operatorname{Accuracy}\left(y_{i}\right)} y_{i}, \quad i=1,2$.

In our case let accuracy $\left(w_{i}\right)=3$

$\xi_{1}=1000 y_{1}, \quad \xi_{2}=1000 y_{2}$

In this way, the continuous variable is considered as an integer one with 3 digit accuracy.

Then the objective with the new variables is

$u=3 x_{1}+5 x_{2}+\frac{\xi_{1}}{1000}+\frac{\xi_{2}}{500}$,

where now all variables are integers,

$2 x_{1}+x_{2}+\frac{\xi_{1}}{1000}+\frac{\xi_{2}}{500} \leq 4 ; x_{1}+3 x_{2}+\frac{\xi_{1}}{1000}+\frac{\xi_{2}}{1000} \leq 5$;

$x_{1} \geq 0 ; x_{2} \geq 0 ; \quad \xi_{1} \geq 0 ; \quad \xi_{2} \geq 0 ;$

$\left(x_{1}\left|x_{2}\right| \xi_{1} \mid \xi_{2}\right) \in$ Integers

The solution is

$x_{1}=1, x_{2}=1, \xi_{1}=1000, \xi_{2}=0$,

then

$\left\{y_{1}, y_{2}\right\}=10^{-3} \quad\left\{\xi_{1}, \xi_{2}\right\}=\{1,0\}$.

This technique will be employed in Section 4, dealing with the GNSS ambiguity solution.

\section{Computing the next best integer solution}

With ambiguity resolution, one often also would like to be able to compute the next best integer solution for ambiguity validation purposes using e.g. the ratio test, see [14]. Let us illustrate this computation with the problem Eq. (3). The objective function is,

$q=27 x_{1}^{2}-18 x_{1} x_{2}+4 x_{2}^{2}-3 x_{2}$. 
Then the first best integer minimum,

$\left\{x_{1}, x_{2}\right\}=\{1,3\}$.

Introducing a new constrain to avoid this minimum,

$q>q(1,3)$,

we solve the problem again,

$\left\{x_{1}, x_{2}\right\}=\{1,2\}$.

That means, after computing the best solution, we can construct a new constrain, and repeat the minimization to avoid best solution and get the next best integer one.

\section{Solution of GNSS phase ambiguity problem}

In Section 5, the third algorithm with some modifications will be employed since it has turned out that the first and second algorithms can solve only the simple configuration problem [15].

Now let us consider a more serious model configuration. The data are from field measurements, and the theoretical result for the coordinates is the zero vector $\{x, y, z\} \rightarrow\{0,0,0\}$, a base-line solution.

The suggested algorithm is a heuristic one and it does not ensure to find the global integer minimum. However, when this minimum is in the neighborhood of the floating minimum, the method can be very efficient. The flow chart of the algorithm can be seen in Fig. 4.

In this case of Successive Nonlinear Solution for a real satellite configuration we have 10 satellites. One of them is the reference one, and the other 9 are sending signals on two carrier frequencies (L1 and L2). So we have 18 ambiguities and 3 coordinates as unknowns. The actual values

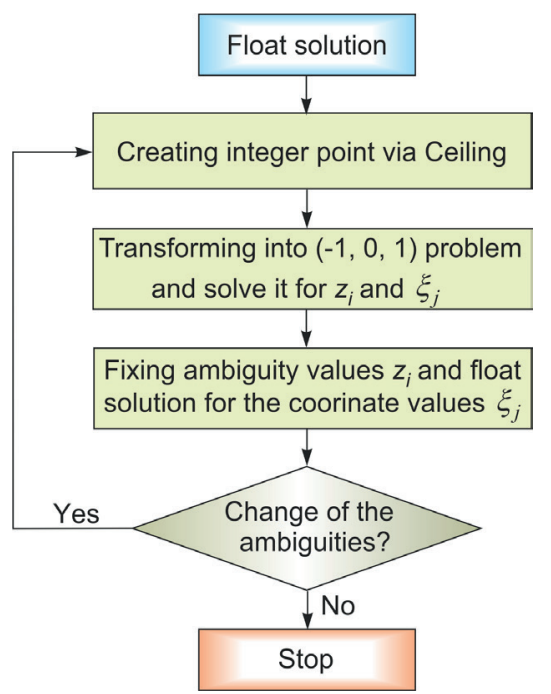

Fig. 4 Flow chart of the nonlinear algorithm
Table 2 Results of the iteration

\begin{tabular}{lccc}
\hline Variables & Float Solution & 1st iteration & 2nd iteration \\
\hline $\mathrm{z}_{1}$ & -25.160 & -25 & -25 \\
$\mathrm{z}_{2}$ & 14.502 & 15 & 15 \\
$\mathrm{z}_{3}$ & 48.192 & 49 & 48 \\
$\mathrm{z}_{4}$ & 0.993 & 1 & 1 \\
$\mathrm{z}_{5}$ & 5.740 & 6 & 6 \\
$\mathrm{z}_{6}$ & -25.403 & -25 & -25 \\
$\mathrm{z}_{7}$ & -25.546 & -25 & -25 \\
$\mathrm{z}_{8}$ & $-22,234$ & -22 & -22 \\
$\mathrm{z}_{9}$ & -65.855 & -65 & -66 \\
$\mathrm{z}_{10}$ & 27.886 & 28 & 28 \\
$\mathrm{z}_{11}$ & 10.614 & 11 & 11 \\
$\mathrm{z}_{12}$ & 20.126 & 20 & 20 \\
$\mathrm{z}_{13}$ & -3.003 & -3 & -3 \\
$\mathrm{z}_{14}$ & -6.218 & -6 & -6 \\
$\mathrm{z}_{15}$ & 12.690 & 13 & 13 \\
$\mathrm{z}_{16}$ & -6.420 & -6 & -6 \\
$\mathrm{z}_{17}$ & 8.823 & 9 & 9 \\
$\mathrm{z}_{18}$ & 8.123 & 8 & 8 \\
\hline$\xi_{1}$ & 13.639 & 7.575 & -3.129 \\
$\xi_{2}$ & -55.421 & -7.564 & 2.466 \\
$\xi_{3}$ & 101.112 & 20.533 & 0.484 \\
Residual & 2.816 & 405.690 & 4.716 \\
\hline & & &
\end{tabular}

of the input arrays were provided by Khodabandeh [10] which can be found in the Appendix.The results of the iteration steps can be seen in Table 2 .

No more iteration is necessary since we get the same result. Applying the LAMBDA method, the same result was achieved [10].

\section{Conclusions}

The algorithms based on local as well as global linearization were proved to be efficient in cases of one carrier frequency. The third one, a locally nonlinear, iterative algorithm, can be employed successfully when L1 and L2 carrier frequencies are used with weighting matrix having elements of very different magnitudes. For multi-GNSS cases, when more satellite should be tracked simultaneously, one may employ the same strategy however at this time the memory management of CAS is not allow to handles large system of equations.

\section{Acknowledgement}

The authors are grateful to Amir Khodabandeh for data and collaboration (University of Melbourne) as well as Joseph Awange (School of Spatial Sciences of the Curtin 
University, Perth) and Daniel Lichtblau (Wolfram Mathematica, Urbana-Champaign, Illinois, USA). In addition the authors are also grateful to professors Peiliang Xu, Kyoto University and Alfred Leick, University of Maine for their critical but useful remarks which could help to

\section{References}

[1] Rózsa, S. "Modelling Tropospheric Delays Using the Global Surface Meteorological Parameter Model GPT2", Periodica Polytechnica Civil Engineering, 58(4), pp. 301-308, 2014. https://doi.org/10.3311/PPci.7267

[2] Juni, I., Rózsa, S. "Validation of a New Model for the Estimation of Residual Tropospheric Delay Error Under Extreme Weather Conditions", Periodica Polytechnica Civil Engineering, 63(1), pp. 121-129, 2019.

https://doi.org/10.3311/PPci.12132

[3] Leick, A., Rapoport, L., Tatamikov, D. "GPS Satellite Surveying", John Wiley \& Sons, Hoboken, NJ, USA, 2015.

[4] Grafarend, E. W. "Mixed Integer-Real Valued Adjustment (IRA) Problems: GPS Initial Cycle Ambiguity Resolution by Means of the LLL Algorithm", In: Grafarend, E. W., Krumm, F. W., Schwarze, V. S. (eds.) Geodesy - The Challenge of the 3rd Millennium, Springer, Berlin, Heidelberg, Germany, 2003, pp. 311-327. https://doi.org/10.1007/978-3-662-05296-9_32

[5] Teunissen, P. J. G., De Jonge, P. J., Tiberius, C. C. J. M. "Performance of the LAMBDA Method for fast GPS Ambiguity Resolution", Navigation, 44(3), pp. 373-383, 1997. https://doi.org/10.1002/j.2161-4296.1997.tb02355.x

[6] Xu, P., Shi, C., Liu, J. "Integer estimation methods for GPS ambiguity resolution: an applications oriented review and improvement", Survey Review, 44(324), pp. 59-71, 2012.

https://doi.org/10.1179/1752270611Y.0000000004

[7] Teunissen, P. J. G. "Quality control in integrated navigation systems", IEEE Aerospace and Electronic Systems Magazine, 5(7), pp. 35-41, 1990. https://doi.org/10.1109/62.134219

[8] Teunissen, P. J. G. "The least-squares ambiguity decorrelation adjustment: a method for fast GPS integer ambiguity estimation", Journal of Geodesy, 70, pp. 65-82, 1995. improve the paper. The article was completed at the School of Spatial Sciences of the Curtin University, Perth AU during the first author's visit there. This work was partially funded by National Research, Development and Innovation Office - NKFIH No. 124286.

[9] De Jonge, P., Tiberius, C. C. J. M. "The LAMBDA method for integer ambiguity estimation implementation aspects", Publications of the Delft Geodetic Computing Centre, LGR-Series, 12, pp. 1-49, 1996. [pdf] Available at: https://d1rkab7tlqy5f1.cloudfront.net/CiTG/Over $\% 20$ faculteit/Afdelingen/Geoscience $\% 20$ $\% 26 \% 20$ Remote $\% 20$ sensing/Research/Positioning\%2C\%20 Navigation\%20and\%20Timing\%20\%28PNT\%29/GPS/lgr12.pdf [Accessed: 15 December 2019]

[10] Khodabandeh, A. "Result of the LAMBDA method", School of Spatial Sciences of the Curtin University, Perth, (personal communication, 10 April 2018).

[11] Li, D., Sun, X. "Nonlinear Integer Programming", Springer US, New York, NY, USA, 2006. https://doi.org/10.1007/0-387-32995-1

[12] Champton, B., Strzebonski, A. "Constrained Optimization. Wolfram Mathematica Tutorial Collection", Wolfram Research, Inc., Long Hanborough, UK, 2008. [online] Available at: http://www.johnboccio.com/MathematicaTutorials/08_ConstrainedOptimization.pdf [Accessed: 15 December 2019]

[13] Mitsos, A., Chachuat, B., Barton, P. I. "McCormick-Based Relaxations of Algorithms", SIAM Journal on Optimization, 20(2), pp. 573-601, 2009. https://doi.org/10.1137/080717341

[14] Teunissen, P. J. G., Verhagen, S. "On the Foundation of the Popular Ratio Test for GNSS Ambiguity Resolution", In: Proceedings of the 17th International Technical Meeting of the Satellite Divisionof The Institute of Navigation (ION GNSS 2004), Long Beach, CA, USA, 2004, pp. 2529-2540.

[15] Paláncz, B. "Numeric-symbolic solution of GPS phase ambiguity problem with Mathematica", Wolfram Library Archive, Long Hanborough, UK,2018. [online] Available at: http://library.wolfram. com/infocenter/MathSource/9705/ [Accessed: 15 December 2019] 


\section{Appendix}

The matrix $A$ and vector $y$ is,

$A=\left(\begin{array}{rrr}0.11350 & 0.40225 & 0.50828 \\ -1.09230 & -0.16510 & 1.02080 \\ -0.44717 & -0.17185 & -0.40806 \\ -0.81536 & -0.47265 & -0.13043 \\ -0.37498 & 0.88919 & 1.02100 \\ -0.28402 & 0.51891 & 1.08210 \\ -0.63514 & -0.23136 & 0.96756 \\ -1.59760 & -0.14047 & 0.59082 \\ -1.14130 & -0.32446 & -0.30627 \\ 0.11350 & 0.40225 & 0.50828 \\ -1.09230 & -0.16510 & 1.02080 \\ -0.44717 & -0.17185 & -0.40806 \\ -0.81536 & -0.47265 & -0.13043 \\ -0.37498 & 0.88919 & 1.02100 \\ -0.28402 & 0.51891 & 1.08210 \\ -0.63514 & -0.23136 & 0.96756 \\ -1.59760 & -0.14047 & 0.59082 \\ -1.14130 & -0.32446 & -0.30627 \\ 0.11350 & 0.40225 & 0.50828 \\ -1.09230 & -0.16510 & 1.02080 \\ -0.44717 & -0.17185 & -0.40806 \\ -0.81536 & -0.47265 & -0.13043 \\ -0.37498 & 0.88919 & 1.02100 \\ -0.28402 & 0.51891 & 1.08210 \\ -0.63514 & -0.23136 & 0.96756 \\ -1.59760 & -0.14047 & 0.59082 \\ -1.14130 & -0.32446 & -0.30627 \\ 0.11350 & 0.40225 & 0.50828 \\ -1.09230 & -0.16510 & 1.02080 \\ -0.44717 & -0.17185 & -0.40806 \\ -0.81536 & -0.47265 & -0.13043 \\ -0.37498 & 0.88919 & 1.02100 \\ -0.28402 & 0.51891 & 1.08210 \\ -0.63514 & -0.23136 & 0.96756 \\ -1.59760 & -0.14047 & 0.59082 \\ -1.14130 & -0.32446 & -0.30627\end{array}\right), \quad y=\left(\begin{array}{r}-4.75710 \\ 2.85700 \\ 9.13270 \\ 0.19081 \\ 1.14110 \\ -4.75720 \\ -4.75910 \\ -4.18520 \\ -12.56000 \\ 6.83820 \\ 2.68960 \\ 4.87710 \\ -0.73147 \\ -1.46970 \\ 3.17590 \\ -1.46590 \\ 2.20040 \\ 1.95520 \\ -0.70882 \\ 0.01589 \\ 0.27637 \\ -0.08741 \\ 0.18193 \\ -0.15592 \\ -0.15185 \\ -0.09746 \\ -0.34652 \\ -0.18282 \\ -0.27811 \\ -0.77963 \\ -0.61641 \\ -0.34707 \\ -0.03891 \\ -0.09485 \\ -0.27346 \\ 0.17948\end{array}\right)$

The structure of matrix B is,

$$
B=\left(\begin{array}{cc}
R_{1} & 0 \\
0 & R_{2} \\
0 & 0 \\
0 & 0
\end{array}\right),
$$

where

$R_{1}=\left(\begin{array}{ccccccccc}0.19 & 0 & 0 & 0 & 0 & 0 & 0 & 0 & 0 \\ 0 & 0.19 & 0 & 0 & 0 & 0 & 0 & 0 & 0 \\ 0 & 0 & 0.19 & 0 & 0 & 0 & 0 & 0 & 0 \\ 0 & 0 & 0 & 0.19 & 0 & 0 & 0 & 0 & 0 \\ 0 & 0 & 0 & 0 & 0.19 & 0 & 0 & 0 & 0 \\ 0 & 0 & 0 & 0 & 0 & 0.19 & 0 & 0 & 0 \\ 0 & 0 & 0 & 0 & 0 & 0 & 0.19 & 0 & 0 \\ 0 & 0 & 0 & 0 & 0 & 0 & 0 & 0.19 & 0 \\ 0 & 0 & 0 & 0 & 0 & 0 & 0 & 0 & 0.19\end{array}\right)$

$R_{2}=\left(\begin{array}{ccccccccc}0.24 & 0 & 0 & 0 & 0 & 0 & 0 & 0 & 0 \\ 0 & 0.24 & 0 & 0 & 0 & 0 & 0 & 0 & 0 \\ 0 & 0 & 0.24 & 0 & 0 & 0 & 0 & 0 & 0 \\ 0 & 0 & 0 & 0.24 & 0 & 0 & 0 & 0 & 0 \\ 0 & 0 & 0 & 0 & 0.24 & 0 & 0 & 0 & 0 \\ 0 & 0 & 0 & 0 & 0 & 0.24 & 0 & 0 & 0 \\ 0 & 0 & 0 & 0 & 0 & 0 & 0.24 & 0 & 0 \\ 0 & 0 & 0 & 0 & 0 & 0 & 0 & 0.24 & 0 \\ 0 & 0 & 0 & 0 & 0 & 0 & 0 & 0 & 0.24\end{array}\right)$

The structure of matrix $Q_{y}$ is,

$Q_{y}=\left(\begin{array}{cccc}R_{3} & 0 & 0 & 0 \\ 0 & R_{3} & 0 & 0 \\ 0 & 0 & R_{4} & 0 \\ 0 & 0 & 0 & R_{4}\end{array}\right) 10^{-4}$

where

$R_{3}=\left(\begin{array}{lllllllll}0.59566 & 0.30044 & 0.30044 & 0.30044 & 0.30044 & 0.30044 & 0.30044 & 0.30044 & 0.30044 \\ 0.30044 & 0.43245 & 0.30044 & 0.30044 & 0.30044 & 0.30044 & 0.30044 & 0.30044 & 0.30044 \\ 0.30044 & 0.30044 & 1.43750 & 0.30044 & 0.30044 & 0.30044 & 0.30044 & 0.30044 & 0.30044 \\ 0.30044 & 0.30044 & 0.30044 & 0.61351 & 0.30044 & 0.30044 & 0.30044 & 0.30044 & 0.30044 \\ 0.30044 & 0.30044 & 0.30044 & 0.30044 & 1.69020 & 0.30044 & 0.30044 & 0.30044 & 0.30044 \\ 0.30044 & 0.30044 & 0.30044 & 0.30044 & 0.30044 & 0.53146 & 0.30044 & 0.30044 & 0.30044 \\ 0.30044 & 0.30044 & 0.30044 & 0.30044 & 0.30044 & 0.30044 & 0.38545 & 0.30044 & 0.30044 \\ 0.30044 & 0.30044 & 0.30044 & 0.30044 & 0.30044 & 0.30044 & 0.30044 & 0.97401 & 0.30044\end{array}\right)$

$R_{4}=\left(\begin{array}{lllllllll}5956.6 & 3004.4 & 3004.4 & 3004.4 & 3004.4 & 3004.4 & 3004.4 & 3004.4 & 3004.4 \\ 3004.4 & 4324.5 & 3004.4 & 3004.4 & 3004.4 & 3004.4 & 3004.4 & 3004.4 & 3004.4 \\ 3004.4 & 3004.4 & 14375 . & 3004.4 & 3004.4 & 3004.4 & 3004.4 & 3004.4 & 3004.4 \\ 3004.4 & 3004.4 & 3004.4 & 6135.1 & 3004.4 & 3004.4 & 3004.4 & 3004.4 & 3004.4 \\ 3004.4 & 3004.4 & 3004.4 & 3004.4 & 16902 . & 3004.4 & 3004.4 & 3004.4 & 3004.4 \\ 3004.4 & 3004.4 & 3004.4 & 3004.4 & 3004.4 & 5314.6 & 3004.4 & 3004.4 & 3004.4 \\ 3004.4 & 3004.4 & 3004.4 & 3004.4 & 3004.4 & 3004.4 & 3854.5 & 3004.4 & 3004.4 \\ 3004.4 & 3004.4 & 3004.4 & 3004.4 & 3004.4 & 3004.4 & 3004.4 & 9740.1 & 3004.4 \\ 3004.4 & 3004.4 & 3004.4 & 3004.4 & 3004.4 & 3004.4 & 3004.4 & 3004.4 & 28100 .\end{array}\right)$

